\title{
ADAPTIVE TECHNIQUE FOR THE MINIMIZATION OF EOG ARTEFAGTS FROM EEG SIGNALS USING TDL STRUCTURE AND NONLINEAR, ESTIMATION MODEL
}

\author{
P. K. Sadasivan and D. Narayana Dutt \\ Department of Electrical Communication Engg. \\ Indian Institute of Science \\ Bangalore-560 012, INDIA
}

\begin{abstract}
EEG records are often contaminated with extracerebral signals called artefacts and one of the main disturbances is due to eye movements which generate an electrical activity called EOG. In this paper, we use an adaptive noise cancellation scheme in a novel way for the minimization of the EOG artefacts from corrupted EEG signals. This method is based on the fact that the transfer function of the biologcal neuron can be modelled as a sigmoidal nonlinearity. Comparison of the time plots as also the smoothed linear prediction spect :a show that the proposed method effectively minimize!: the EOG artefacts from corrupted EEG signals.
\end{abstract}

\section{Introduction}

Electroencephalogram (EEG) is the electrical activity of the brain and it contains diagnostic information on various neurological conditions. These signals reflect the activities in underlying brain structure and particularly in the cerebral cortex below the scalp surface. EEG signals are measured from electrodes placed on the scalp, and are often very small in amplitude. EEG has become an indispensable tool In clinical neurophysiology and related fields. EEG signals mainly contain four frequency related activities. The most obvious activity is a rhythmic activity called the alpha activity, and is by definition within the frequency range of $8-\mathbf{1 3} \mathbf{~ H r}$. The rhythmic activity in the higher frequency range of $14-30 \mathrm{~Hz}$ is called beta activity and its spatial distribution is different from that of alpha activity Lower frequency activities are called delta activity in the frequency range of $0.5-3 \mathrm{~Hz}$ and theta activity in the frequency range of $4-7 \mathrm{~Hz}$. In addition to these activities, EEG also contains transients like spikes, spindles etc [1].

It is well known that EEG signals are often seriously contaminated with extracerebral signals called artefacts. Artefacts are caused by sources bath internal and external to the body. Artefacts reduce the clinical usefulness of EEG signals and make both manual and automatic analysis difficult or, in some cases, impossible because of the similarity between artefacts and the signals of interest. In electroencephalography, the presence of ocular artefacts which results from eye movements and blinks are constant source of difficultyin distinguishing normal activities from abnormal ones. Blinking or moving the eyes produces large electrical potentials (in $m V$ range) around the eyes called the electrooculogram (EOG). The sources of these potentials is the corneo-retinal dipole and short circuiting of it by the eyelids [2][3]. EOG spreads across the head or scalp to contaminate the EEG signals and this artefact severely affects the performance of the automated EEG processing systems. Effective elimination of EOG artefacts from the collected EEG data is an essential step in preparing EEG data for further analysis. Hence the removal of EOG signals forms an important part of the computer processing of EEG signals.

Several methods have been proposed for removing or controlling ocular artefacts from contaminated EEG signals. Rejection method is the simplest method in which the ocular artefact is controlled by discarding the EEG segments that have artefacts due to eye movements [4]. This can result in the loss of considerable and sometimes significant portions of the data and rejection of these sections could make the data even unrepresentative [5][6]. Rejection has also been achieved in the frequency domain [7]. To reduce the amount of data lost by the rejection method, the subjects are often asked to follow the eye fixation method [8]. EOG subtraction method has been reported by various authors. A method suggested by Girton and Kamiya [9] subtracts a percentage of the horizontal and vertical EOG's from the time domain EEG signals. Corhy and Kopell[10] and Hillyard and Galambos [11] have suggested offline methods where eye movements and eye blinks are individually separated and removed with different fractions of the EOG's. Verleger et. al. [5] computed the eye movement artefact by applying the method of least squares to one EOG channel and subsequently subtracted it from the corrupted EEG signal to get EOG free EEG signal. Fortgens and De Bruin [12] also applieu the method of least squares with EOG recorded from four locations. Later Jervis et al. reported that these two methods are identical [13] and they 
result in distortion of EEG responses [14]. Whitton et al. [15] and Woestenburg et al. [16] have described spectral methods for removing ocular artefacts. Barlow and Remond [17] described a more sophisticated on-line correction technique. Quilter et al. [18] have described an off-line ocular artefact removal procedure which involves cross- correlating the EOG chanriels with the EEG channels. The technique by Quilter et al. has been extended by Jervis et al. [13] [19] to three and four parameter models. McCallum and Walter [20] had suggested an analog on-line correction technique for EOG artefact minimization from corrupted EEG signals.

In order to remove the EOG artefact from corrupted EEG signals, filters which are either fixed or adaptive may be used. An adaptive filter has the advantage that it can adjust its own parameters automatically. Adaptive noise cancellation (ANC) [21] is a technique which uses an adaptive filter to process the reference noise in order to estimate the noise that has corrupted some signal. The corrupted signal is the primary input to the ANC scheme. The estimated noise is then subtracted from the primary signal, reducing or cancelling the noise from the corrupted signal. Adaptive filters are self optimizing in that they adjust their parameters so as to cancel out the maximum amount of noise even as the statistics of the reference noise or the primary signal change. Jervis et al. [22] have developed a microprocessor based ocular artefact remover based on these ideas. Ifeacher et al. [23] have suggested a recursive least squares (RLS) algorithm with exponential data weighting for the on-line removal of ocular artefact from corrupted EEG signals. A knowledge based enhancement of human EEG signals has also been reported by Ifeacher et al. [24]. Reduction of eye movement artefact from corrupted EEG signals has also been achieved by adaptive filters with fast RLS algorithm [25].

In this paper, we are using an ANC technique in a novel way for the minimization of EOG artefact from corrupted EEG signals. This method is based on the knowledge of the transfer function of the biological neuron, which has been modelled as a sigmoidal nonlinearity. In the ANC technique that we have used here, the corrupted EEG signal recorded from $F p_{1}$ position on the scalp forms the primary input and the two channels of EOG signals from left and right. eye positions form the two reference inputs on which the nonlinear estimation scheme bas been applied to get an estimate of EOG artefact. The parameters involved in the estimation are updated sample by sample using Widrow-Hoff least mean squares algorithm [21]. A comparison of the time plots as also the smoothed LP spectra show that the proposed algorithm effectively minimizes the EOG artefacts from corrupted EEG signals.

\section{Problem Formulation}

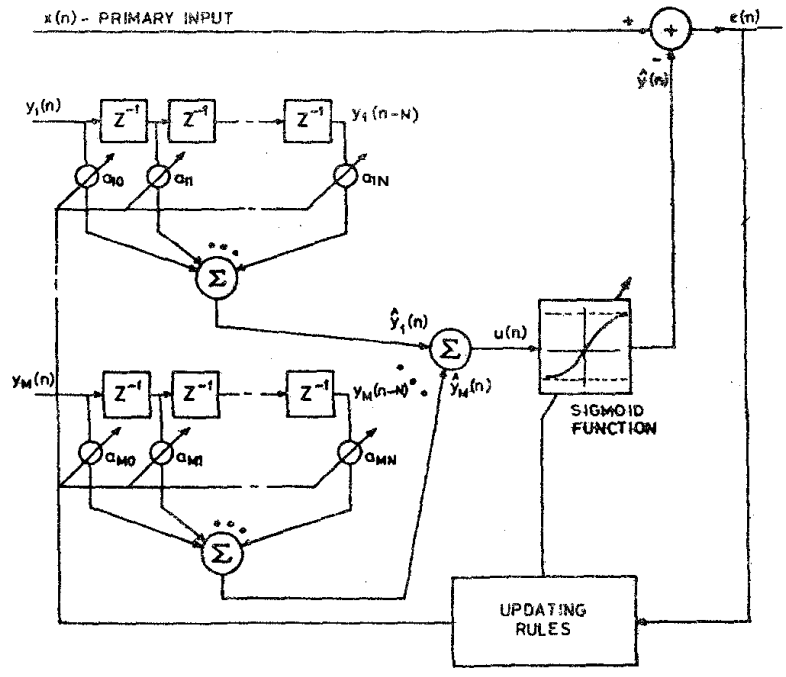

Fig.I Block diagram of the proposed Algorithm.

Let $x(n)$ be the primary input and $y_{1}(n), y_{2}(n) \ldots y_{M}(n)$

be the $M$ reference inputs to the ANC.

The estimated value of the $n^{\text {th }}$ sample of the $i^{\text {th }}$ reference input is given by,

$$
\hat{y}_{i}(n)=\mathbf{Y}_{i}^{T}(n) \mathbf{A}_{i}(n)
$$

where

$$
\mathbf{Y}_{i}(n)=\left[\begin{array}{llll}
y_{i}(n) & y_{i}(n-1) & \cdots & y_{i}(n-N)
\end{array}\right]^{T}
$$

is the $i^{\text {th }}$ reference input vector of $\operatorname{size}(N+1) \times 1$

$$
\mathbf{A}_{i}(n)=\left[\begin{array}{llll}
a_{i 0}(n) & a_{i 1}(n) & \cdots & a_{i N}(n)
\end{array}\right]^{T}
$$

is the $i^{\text {th }}$ filter coefficient vector of size $(N+1) \times \mathrm{i}$, and $N$ is the number of delay units used in each reference input TDL structure.

The input to the sigmoidal nonlinearity, $u(n)$ is given by

$$
u(n)=\sum_{i=1}^{M} \hat{y}_{i}(n)
$$

The filtered output $\hat{y}(n)$ is obtained by passing $u(n)$ through sigmoidal nonlinearity and is given by

$$
\hat{y}(n)=\frac{:}{1+\exp \left(\frac{-u(n)}{\lambda(n)}\right)}
$$

$\hat{y}(n)$ is a function of $:$ and $\lambda$, where $\lambda$ determin the slops nonlinearity at $\hat{y} n=b$

The objective is to find the cotimiz values of the hit.

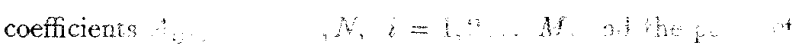

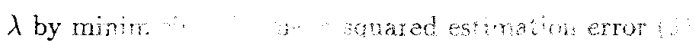

$$
\left.F \cdot{ }^{2}(n)\right]
$$


where

$$
e(n)=x(n)-\hat{y}(n)
$$

is the estimation error and $E[\cdot]$ is the expectation operator.

Applying Widrow-Boff least mean squares technique for updating the parameters involved in the algorithm, we get the following update equations.

1. The sigmoidal nonlir earity coefficient:

$$
\lambda(n+1)=\lambda(n)-\left.\mu \frac{\partial J}{\partial \lambda}\right|_{\lambda=\lambda(n)}
$$

which results in

$$
\lambda(n+1)=\lambda(n)-\frac{2 \mu e(n) u(n) \exp \left(\frac{-u(n)}{\lambda(n)}\right)}{\left\{\lambda(n)\left[1+\exp \left(\frac{-u(n)}{\lambda(n)}\right)\right]\right\}^{2}}
$$

2. The filter coefficients:

$$
\begin{aligned}
a_{k l}(n+1)=a_{k l}(n)-\mu \frac{\partial J}{\partial a_{k l}} & \left.\right|_{a_{k l}=a_{k l}(n)} \\
k=1, \ldots, M, \quad l=1, \ldots, N . &
\end{aligned}
$$

which results in

$$
a_{k l}(n+1)=a_{k l}(n)+\frac{2 \mu e(n) y_{k}(n-l) \exp \left(\frac{-u(n)}{\lambda(n)}\right)}{\lambda(n)\left[1+\exp \left(\frac{-u(n)}{\lambda(n)}\right)\right]^{2}}
$$

where $\mu$ is a positive constant which controls the convergence rate of the algorithm.

Summarising, the different steps in the algorithm are

1. Calculate $\hat{y}(n)$.

2. Calculate the error, $e(n)$.

3. Update the parameters.

\section{Results and Discussion}

The ANC based artefact minimization scheme, formulated in the previous section, has been studied using computer simulations. The two derivations of the EOG signals, $E O G_{1}$ and $E O G_{2}$ from left and right eye positions were recorded on separate channels simultaneously with the EOG corrupted EEG signal from $F p_{1}$ position on the scalp using a Nihonkhoden EEG machine. These signals were digitized at $100 \mathrm{~Hz}$ and then low pass filtered at $35 \mathrm{~Hz}$ using a linear phase finite impulse response digital filter. The corrupted EEG signal has been used as the primary input to the ANC and the two derivations of the EOG signals as the reference inputs. Fig.2a shows the EOG corrupted EEG signal in which the low frequency high amplitude activity is due to the eye movements. Fig. $2 b$ is the plot of the corrected EEG signal from which the EOG has been effectively minimized with the proposed algorithm. It may be observed from Fig. $2 \mathrm{~b}$ that the EOG artefact reduction is not that good in the beginning portion but improves considerably with time. This is because of the time that the adaptive algorithm takes to reach the near-optimum values of the parameters.

In order to make an assessment of the effectiveness in removing the EOG artefact from corrupted EEG signals using the proposed algorithm, we have calculated the smoothed power spectrum of these signals using linear prediction [26] technique. Fig.3a shows the smoothed spectrum of the corrupted EEG signal which shows a peaky response at about $2.5 \mathrm{~Hz}$ implying the presence of low frequency eye movement artefact. In Fig. $3 \mathrm{~b}$ (which is the smoothed LP spectrum of the corrected EEG signal) the peaky response at low frequency is being effectively reduced which implies that the proposed algorithm is very effective in minimizing the EOG artefart from corrupted EEG signals.

\section{Conclusions}

We have used the ANC scheme in a novel way for the minimization of EOG artefacts from corrupted EEG signals. The nonlinearity in the noise estimation is introduced using a sigmoidal function, which is the transfer function of the biological neuron. Widrow-Hoff least mean square technique is used for updating the parameters involved in the algorithm. Comparison of the time plots and the smoothed LP spectrum show that the proposed method works well in minimizing EOG artefacts from corrupted EEG signals.

\section{References}

[1 ] Storm Van Leeuwen, W., Bickford, R., Brazier, M., Cobb, W.A., Dondey, H., Gastaut, H., Gloor, P., Henry, C.E., Hess, R., Knott, J.R., Kugler, J., Lairy, G.C., Loeb, C., Magnus, O., Daurella, L.O., Petsche, N., Schwab, R., Walter, W.G., and Widen, L., "Proposal for an EEG terminology by terminology committee of the International federation for Electroencephalography and Clinical Neurophysiology", Electro. Clin. Neurophysiol., Vol.20, 1966, pp.306-310.

[2] Geddes, L.A and Baker, L.E, Principles of applied biomedical instrumentation. Wiley and Sons, New York, 1975, pp.509-517.

[3 ] Hillyard, S.A, "Methodological issues in CNV research" ${ }^{\mathrm{n}}$, In Bioelectric Recording Techniques Part B. Electro. and Human Brain Potentials, Thomson, R.F and Patterson, M.M (Eds.), Academic Press, 1974, pp.259-280. 
[4 ] Barlow, J.S. "Computerized clinical electroencephalography in perspective", IEEE Trans. BME, Vol.26, No:7, 1979, p.p.377-391.

[5 ] Verleger, R., Gasser, T. and Mocks, J., "Correction of EOG artefacts in event related potentials of the EEG: aspects of reliability and validity", Psychophysiology, Vol.19, 1982, pp.472480 .

[6 ] Gratton, G., Coles, M.G.H. and Donchin, E., "A new method of off- line removal of ocular artefacts", Electro. Clin. Neurophysiol., Vol.55, 1983, pp. 468-484.

[7 ] Gevins, A.S., Yeager, C.L., Zeitlin, G.M., Ancoli, S and Dedon, M.F., "On-line computer rejection of EEG artefact" Electro. Clin. Neurophysiol., Vol.42, 1977, pp. 267-274.

[8 ] Papakostopoulos, D., Winter, A. and Newton, P., "New techniques for the control of eye potential artefacts in multichannel CNV recordings", Electro. Clin. Neurophysiol., Vol.34, 1973, pp. 651-653.

[9 ] Girton, D.G and Kamiya, J., "A simple on-line technique for removing eye movement artefacts from the EEG." Electro. Clin. Neurophysiol., Vol.34, 1973, pp. 212-216.

[10 ] Corby, J.C and Kopell, B.S. "Differential contributions of blinks and vertical eye movements as artefacts in EEG recording”, Psychophysiology, Vo1.9, 1972, pp. 640-644.

[11 ] Hillyard, D.G and Galambose, R. "Eye movement artefact in the CNV", Electro. Clin. Neurophysiol., Vol.28, 1970, pp. 173-182.

[12] Fortgens, C and De Bruin, M.P., "Removal of eye movement and ECG artefacts from non-cephalic reference EEG", Electro. Clin. Neurophysiol., Vol.56, 1983, pp. 90-96.

[13 ] Jervis, B.W., Nichols, M.J., Allen, E.M., Hudson, N.R. and Johnson, T.E., "The assessment of two methods for removing eye movement artefact from EEG", Electro. Clin. Neurophysiol., Vol.61, 1985, pp. 444-452.

[14 ] Jervis, B.W., Coelho, M. and Morgan, C.W., "Effect on EEG responses of removing ocular artefacts by proportional EOG subtraction", Med. and Biol. Engg. and Computing, Vol.27, 1989, pp. 484-490.

[15 ] Whitton, J.L., Leu, F. and Moldofsky, H ., "A spectral method for removing eye movement artefacts from the EEG", Electro. Clin. Neurophysiol., Vol.44, 1978, pp. 735-741
[16 ] Woestenburg, J.C., Verbaten, M.N. and Slagen, J.L., "Removal of eye movement artefact from the EEG by regression analysis in the frequency domain”, Biol. Psychol. Vol.16, 1983, pp. 127-147.

[17] Barlow, J.S and Remond, A. "Eye movement artefact nulling in EEG's by multichannel on-line EOG subtraction", Electro. Clin. Neurophysiol., Vol.55, 1981, pp. 418-423.

[18 ] Quilter, P.M., MacGillivray, B.B. and Wadbrook, D.G., "The removal of eye movement artefact from EEG signals using correlation technique, In random signal analysis", IEE Conf. Publ., Vo1.159, 1977, pp. 93-100.

[19 ] Jervis, B.W., Nichols, M.J., Allen, E.M., Hudson, N.R. and Johnson, T.E., "The quantitative assessment of electroencephalograms corrected for eye movement artefacts", 1st Europ. Signal Proc. Conf., Lausanne, Switzerland, 1980.

[20 ] McCallum, W.C and Walter, W.G. "The effects of attention and distraction on the contingent negetive variation in normal and neurotic subjects", Electro. Clin. Neurophysiol., Vol.25, 1968, pp. 319-329.

[21 ] Widrow, B., Glover,Jr, J.R., McCool, J.M.. Kaunitz, J., Williams, C.S., Hearn, R.H., Zeidler, J.R., Dong, Jr, E. and Goodlin, R.C., "Adaptive noise cancelling: principles and applications", Proc. IEEE, Vo1.63, 1975, pp.1692-1716.

[22 ] Jervis, B.W., Ifeacher, E.C., Allen, E.M., Morris, E.L. and Hudson, N.R., "Removal of ocular artefact from the human EEG", IEEE 7th Annual Conf. of the Engg. in Med. and Biol. Soceity, Chicago, USA, 1985, pp.101-107.

[23] Ifeacher, E.C., Jervis, B.W., Morris, E.L., Allen, E.M. and Hudson, N.R., "New on-line method for removing ocular artefacts from EEG signals", Med. and Biol. Engg. and Computing, Vol.24, 1986, pp. 356-364.

[24 ] Ifeacher, E.C., Hellyar, M.T., Mapps, D.J. and Allen, E.M., "Knowledge based enhancement of human EEG signals", IEE Proc., V01.137, Pt.F, 1990, pp. 302-310.

[25] Sadasivan, P.K and Narayana Dutt, D., "Eyemovement artefact rejection in EEG signals by adaptive cancellation technique", Proc, National Conf, on Elect Circuits and Systems, Roorkee, 1989, pp. 254-256.

[26] Makhoul, J., "Linear prediction: A tutorial review", Proc IEEE, Vol.3, 1975, pp. 561-580. 

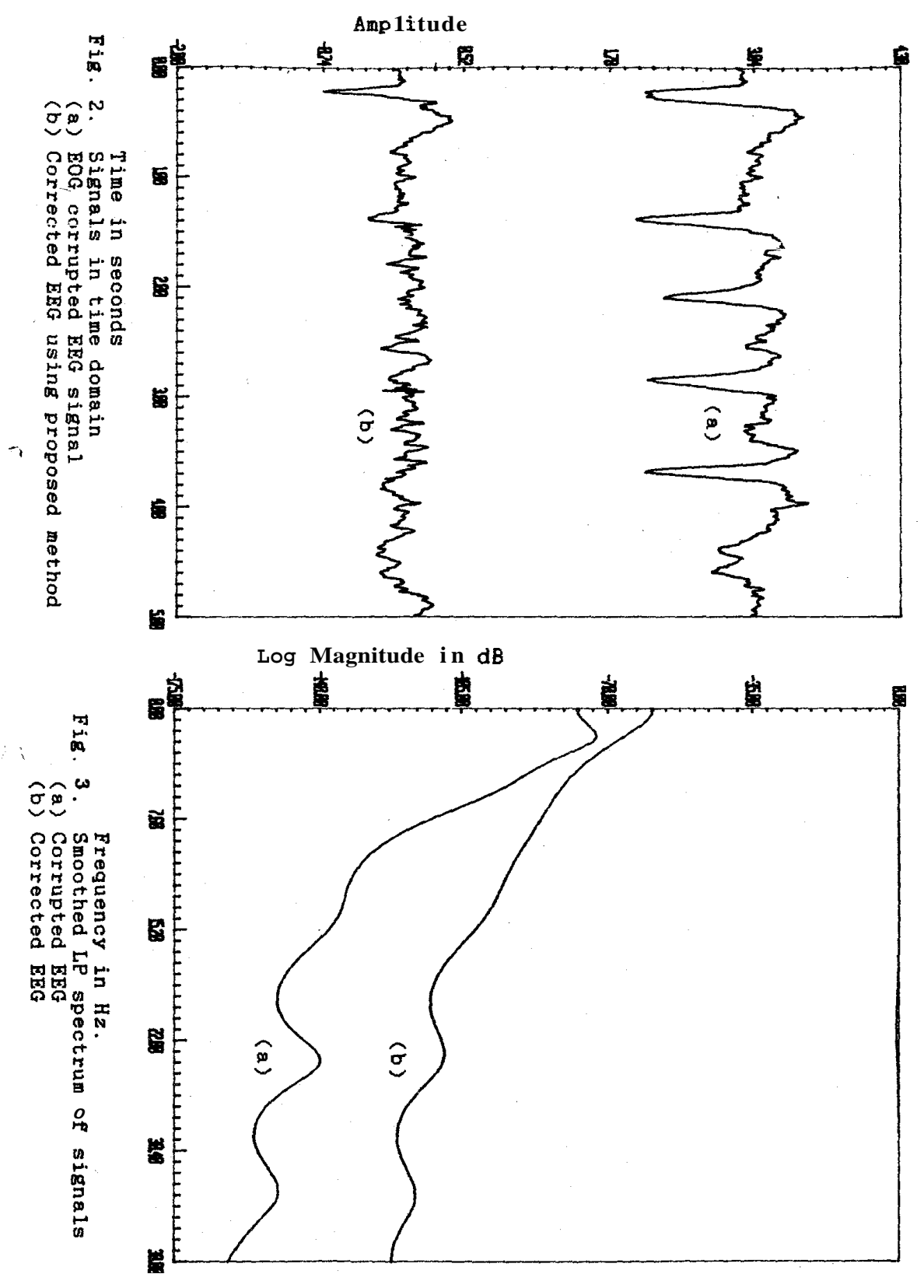